\section{Another reason for saving Sakharov}

SIR-I believe it is crucial in our attempts to rescue Andrei Sakharov to reemphasize Sakharov's scientific legacy to the scientific community at large and to Soviet officials in particular. Studying recently an early paper on gravitation theory by Sakharov'. I realized that this work not only provides a new way of looking at gravity, but also gives us a new weapon in the battle for Sakharov's life. The paper, which describes the possible connection between gravitation and vacuum fluctuations, has been referred to in a number of major textbooks ${ }^{2-1}$. My own investigations have given me an even higher opinion of this seminal paper.

I have been studying the influence of a suggested inhomogeneity in zero-point vacuum fluctuations on free particle motion and have come to the conclusion ${ }^{5 . n}$ that in the general case the particle worldlines become geodesics of a Riemannian geometry. So I was able to appreciate Sakharov's main suggestion of a direct connection between the vacuum fluctuations and gravity (that is, Riemannian geometry of spacetime). In the appropriate sense, Sakharov obtains the Hilbert Lagrangian of general relativity. As a result the Einstein equations acquire the meaning of connection between an inhomogeneity in the vacuum fluctuations and the distribution of matter. Some formal difficulties in the development of Sakharov's concept are removed by the recently derived covariant description of gravitational energy.

I believe that Sakharov's concept may resolve the 60-year confrontation between quantum field theory approaches to gravity and Einstein's general relativity, after hundreds of futile attempts to bridge this gap. Once some formal difficulties are removed, I anticipate the development of the physics of vacuum fluctuations as a fundamental branch of science with many applications. Hence I believe that historically Sakharov's name will be among the most illustrious names in science. Like Evariste Galois, another genius of the highest originality, Sakharov chose the way dictated by his moral self-obligation and this deprived him of the opportunity to advance many of his ideas. Like Galois, he gave only a short synopsis of his concept. I believe that the concept will steadily increase in importance to science, as did Galois' contribution.

Hence I perceive a new starting point in our efforts to save Sakharov's life. His humanitarian activities have been completely rejected by Soviet authorities as part of the dangerous "bourgeois ideology" with which they claim no conciliation is possible. They do not think that Sakharov's role in the creation of Soviet nuclear weapons is worthy of reward or respect in the West. He is not considered as a head of some scientific school inside the Soviet Union or abroad. So, to Soviet authorities. Sakharov is nothing but a Western tool of the cold war. They assume that he will be forgotten in the West as soon as he is out of sight. Hence, from their point of view. Sakharov may safely be ignored. Perhaps the new Soviet leaders will rectify this situation when they realize that his scientific legacy will not be forgotten by history and that Sakharov's persecution will be recalled in every physics textbook.

It is important to make it clear to the Soviet authorities (and perhaps to some of us also) that Sakharov is likely to be seen as the founder of a new fundamental branch of physics which may find wide application. I believe that the best way to advance this understanding is the rapid development of Sakharov's concept and its broad explanation. My belief is that the investment of money and intellectual resources in Sakharov's concept are completely justified by the expected scientific results. But there is an additional justification. The rescue of Sakharov is our ticket of admission to the human race.

Washington University,

Erast B. Gliner

\section{St Louis, Missouri 631330, USA}

Sakharov, A.D. Doklady Academii Nauk SSSR, 177, 70 (1967) (Translation: Soviet Physics - Doklady 12, 1040 ; $1968)$

2. Misner. C.M.. Thorn. K.S. \& Wheeler, J. A. Gravitation. 427 (Freeman. San Francisco)

Zel'dovich. Ya B. \& Noviklov. I.D. Stars and Relativity. $71-74.77$ (Chicago Press)

Zel'dovich. Ya B. \& Novikov. I.D. The Struchure and Evolution of the Universe, 669 (Chicago) Press).

Gliner E Phys, Rev Lett (submitted)

6. Gliner, E. Quantum Theory, of Fields and Origin of Gravity, Third Loyola Conference on Quantum Theory and Gravitation. (in the press)

\section{Ocean thermal response in man-made climatic change}

SIR-John Woods has raised' important issues concerning the dynamic response of ocean circulation to anthropogenically imposed changes in atmospheric $\mathrm{CO}_{2}$ level. The full implications of the physical characteristics he presented have potentially serious environmental policy consequences beyond those discussed in his paper. The delay in ocean-atmospheric temperature equilibration due to the ocean's thermal mass following the introduction of man-made infrared absorbing trace gases into the atmosphere implies a slower rise in global climatic warming than given by calculations that omit oceanic effects. The time it would take to reach the conventional measure of a significantly hazardous environmental condition (a global temperature rise of about $3^{\circ} \mathrm{C}$, which corresponds to doubling of atmospheric $\mathrm{CO}_{2}$ concentrations to about 600 p.p.m.v.) can thereby be lengthened by from 20 to 100 years ${ }^{2}$.

Woods suggests that such a delay in temperature rise will ease the potential for traumatic climate change by providing extra time for initiation of measures to reduce climatic warming, such as the accelerated development of nuclear in place of fossil energy. But this likely will not be the course of events. Rather, it is more probable that the needed remedial actions will be delayed until the observed climate change can be unequivocally identified with atmospheric $\mathrm{CO}$, growth, by which time its concentration could have risen above a value whose ultimate equilibrium climate effect would be calamitous. From this perspective, thermal inertia of the oceans introduces a type of irreversibility which is obviously of major importance for policy, since global temperatures will continue rising even after a decision to drastically reduce fossil fuel use has been implemented.

Scientists' response to this dilemma is naturally to suggest that predictions, rather than observations of temperature change, be used as the basis for action. But this requires hard political decisions which are made more difficult by the acknowledged lack of understanding and quantifiability of the physical phenomena concerning climatic change and its impacts. How to properly bring the relevant but uncertain scientific information into the decision-making process, is important, and attention is starting to be paid to this type of need ${ }^{2-5}$.

Before we conclude that existence of long thermal ocean inertia lags inevitably results in the major irreversibility dilemma presented above, we should point out a revision in the usual viewpoint that is needed in order to properly address the physics of the problem. The ocean transient response is normally described in terms of an e-folding time for approach to thermal equilibrium - setting atmospheric $\mathrm{CO}_{2}$ level to be constant. However, from a policy viewpoint we need to follow the approach to a steady state following elimination of $\mathrm{CO}$ emissions. All current carbon cycle models predict only a modest fall off rate of $\mathrm{CO}_{2}$ levels following such a step ${ }^{6}$, but these probably do not allow for the involvement of deeper ocean layers that results in the extended e-folding times we have quoted. In fact, it seems intuitively clear than stronger coupling to the deep ocean implies a larger short-term oceanic sink for $\mathrm{CO}$ deposition, and hence a faster fall of atmospheric $\mathrm{CO}_{2}$ concentration following reduction of $\mathrm{CO}_{2}$ emissions. If this qualitative surmise is true (and an analysis of it has yet to be made), the irreversibility feature of the policy issue we have raised may not be as severe as it seems at first glance.

$$
\text { J.A. LAURMANN }
$$

Gas Research Institute,

8600 West Bryn Mawr Avenue,

Chicago, Illinois 60631, USA

1. Woods. J. Nature 314. 578-579 (1985).

2. Hansen, J.E. et al. in Climate Process and Climate Sensitivity (eds Hansen, J.E. \& Takahashi. T.) (American Geophysical Union. Washington DC 1984

Ruckelshaus. W.D. Issues in Science and Technology. National Academy of Sciences 2. 19-38 (1985). 